\title{
Therapy of Venezuelan Patients with Severe Mucocutaneous or Early Lesions of Diffuse Cutaneous Leishmaniasis with a Vaccine Containing Pasteurized Leishmania Promastigotes and Bacillus Calmette-Guerin - Preliminary Report
}

\author{
Jacinto Convit/ ${ }^{+}$, Marian Ulrich, María Argelia Polegre, Angela Avila, Noris Rodríguez, \\ Maria Ilina Mazzedo, Belkis Blanco
}

Instituto de Biomedicina, Universidad Central de Venezuela, Esquina San Nicolás, San José, Apartado Postal 4043, Caracas 1010A, Venezuela

Severe mucocutaneous (MCL) and diffuse (DCL) forms of American cutaneous leishmaniasis (ACL) are infrequent in Venezuela. Chemotherapy produces only transitory remission in DCL, and occasional treatment failures are observed in MCL. We have evaluated therapy with an experimental vaccine in patients with severe leishmaniasis. Four patients with MCL and 3 with early DCL were treated with monthly intradermal injections of a vaccine containing promastigotes of Leishmania (Viannia) braziliensis killed by pasteurization and viable Bacillus CalmetteGuerin. Clinical and immunological responses were evaluated. Integrity of protein constituents in extracts of pasteurized promastigotes was evaluated by gel electrophoresis. Complete remission of lesions occurred after 5-9 injections in patients with MCL or 7-10 injections in patients with early DCL. DCL patients developed positive leishmanin skin reactions, average size $18.7 \mathrm{~mm}$. All have been free of active lesions for at least 10 months. Adverse effects of the vaccine were limited to local reactivity to BCG at the injection sites and fever in 2 patients. Extracts of pasteurized and fresh promastigotes did not reveal differences in the integrity of protein components detectable by gel electrophoresis. Immunotherapy with this modified vaccine offers an effective, safe option for the treatment of patients who do not respond to immunotherapy with vaccine containing autoclaved parasites or to chemotherapy

Key words: immunotherapy - diffuse cutaneous leishmaniasis - mucocutaneous leishmaniasis

American cutaneous leishmaniasis (ACL), a granulomatous protozoan infection caused by several species of Leishmania, is a zoonosis which is maintained in the wild by rodents and other hosts. The infection is transmitted to humans by sand fly vectors of the Lutzomyia genus in the New World. Though precise data are scarce, many thousands of humans are infected throughout Central and South America (Grimaldi et al. 1989).

ACL presents a spectrum of clinical manifestations in humans (Convit et al. 1993). Localized cutaneous leishmaniasis (LCL) is characterized by one or more ulcers with a limited tendency to self-healing; patients develop antigen-specific cell-mediated immunity to the microorganism. In contrast, progressive diffuse cutaneous leishmaniasis (DCL) is characterized by the presence of numerous nodular lesions and an antigen-specific absence of manifestations of delayed hypersensitivity and protective immunity. These presentations appear to correspond to polar forms of cutaneous leishmaniasis, similar to those described in different strains of inbred mice (Reiner \&

Financial support: Scientific Initiative of the Millennium Project, grant 4572-VE, World Bank/Government of Venezuela ${ }^{+}$Corresponding author. Fax: $+58-212-8611258$. E-mail: jconvi@telcel.net.ve

Received 7 July 2003

Accepted 12 January 2004
Locksley 1993). We consider diverse manifestations of ACL such as extensive vegetative and verrucous cutaneous lesions, disseminated cutaneous leishmaniasis, and mucocutaneous leishmaniasis (MCL) to be clinical presentations of intermediate cutaneous leishmaniasis (ICL) in this spectrum. While these diverse clinical manifestations reflect fundamental differences in the host's immune response to the infection, variable pathogenic potential of different species of New World Leishmania infecting humans has also been reported. MCL and other forms of ICL in Venezuela are associated with infection by species of the Leishmania (Viannia) braziliensis complex, while DCL is caused by L. (Leishmania) amazonensis and, less frequently, other species of the $L$. (L.) mexicana complex (Convit et al. 1993). Both L. (L.) amazonensis and L. (V.) braziliensis have been identified in cases of disseminated cutaneous leishmaniasis (Carvalho et al. 1994).

Some chronic manifestations of leishmaniasis respond very poorly to conventional chemotherapy. MCL usually responds to conventional treatment, but refractory cases have been observed. MCL can be incapacitating and disfiguring; occasionally it becomes life-threatening when lesions of the pharynx and larynx obstruct the respiratory passages and or cause difficulty in swallowing. Treatment with antimonials or other chemotherapy produces only transitory remissions in the inexorable progression of DCL, even when combined with gamma interferon immunotherapy in an effort to induce Th1 immunological conditions (Salaiza-Suazo et al. 1999). 
More than a decade ago, we reported the use of immunotherapy (IT) with a combined vaccine containing autoclaved $L$. (L.) amazonensis promastigotes and viable Bacillus Calmette-Guerin (BCG) in the treatment of LCL (Convit et al. 1987, 1989). Since these early studies, more than 11,500 Venezuelan patients with LCL have been treated with this form of IT, with clinical healing in more than 95\% (Convit et al. 2003). As reported previously (Convit et al. 1989), a significant number of small groups of patients with MCL and DCL showed clinical improvement with the vaccine containing autoclaved promastigotes when chemotherapy was included in the therapeutic regime, but immunotherapy alone was not evaluated in these groups.

In this study, we report the preliminary results of the use of a modified form of this vaccine, using pasteurized $L$. (V.) braziliensis promastigotes and viable BCG, in the treatment of a small number of patients with refractory MCL and in early cases of DCL. This modified vaccine induced persistent clinical remission of disease and immunological changes which suggest effective immunomodulation without the simultaneous administration of chemotherapy.

\section{PATIENTS AND METHODS}

Patients - Four patients with severe MCL and three with early DCL, evolution of 1 to 5 years, were treated on a compassionate need basis in this study, based on their inadequate response to earlier treatment or clinical presentation associated with an unresponsive form (DCL) of the disease. Leishmaniasis was diagnosed in the Leishmaniasis Clinical Section of the Institute of Biomedicine by clinical, histopathological, immunological, and epidemiological criteria, including the presence of characteristic lesions, granulomatous reactions to living agents and, in the patients with MCL, positive leishmanin reactions. Parasitological confirmation based on the identification of Leishmania in smears or biopsies was possible in 6 of the 7 patients; the species were not identified in this study. Relevant characteristics of these patients are given in Table I. Three patients were treatment failures with meglumine antimoniate chemotherapy. Chemotherapy was suspended in a fourth patient because of severe adverse reactivity to the antimonial after two 20-day cycles, and the three remaining patients were not considered candidates for antimonial treatment because of age or very poor state of health. These patients or their legal guardians gave informed consent after explanation of the protocol, which had been previously approved by the Ethics Committee of the Institute of Biomedicine and the Bio-Ethical Committee of the Venezuelan National Foundation for Scientific and Technological Investigation.

Brief summaries of the pathology and histopathology of these patients are as follows:

Case 1. Extensive infiltrated lesions in nasal fossa, perforation of the cutaneous portion of the septum. Marked infiltration of the palate, uvula, pharynx, left tonsillar fossa. Granuloma with epithelioid foci and scarce intracellular leishmanias. Lesions produced difficulty in swallowing, changes in timbre of voice.
TABLE I

Seven patients with severe mucocutaneous or early diffuse cutaneous leishmaniasis treated with pasteurized vaccine

\begin{tabular}{llccccl}
\hline $\begin{array}{c}\text { Case Clinical } \\
\text { form }\end{array}$ & $\begin{array}{c}\text { Age } \\
\text { (years) }\end{array}$ & $\begin{array}{c}\text { Sex Evolution } \\
\text { (years) }\end{array}$ & $\begin{array}{c}\text { Initial } \\
\text { leishmanin }\end{array}$ & $\begin{array}{l}\text { Previous } \\
\text { treatment }\end{array}$ \\
\hline 1 & MCL & 62 & $\mathrm{M}$ & 4 & $21 \mathrm{~mm}$ & None \\
2 & MCL & 45 & $\mathrm{M}$ & 1 & $28 \mathrm{~mm}$ & 4 cycles CT $b$ \\
3 & MCL & 53 & $\mathrm{M}$ & 4 & $21 \mathrm{~mm}$ & 2 cycles CT \\
4 & MCL & 43 & $\mathrm{~F}$ & 3 & $63 \mathrm{~mm}$ & None \\
5 & DCL & 6 & $\mathrm{M}$ & 5 & $0 \mathrm{~mm}$ & 10 cycles CT \\
6 & DCL & 10 & $\mathrm{~F}$ & 1.5 & $0 \mathrm{~mm}$ & 3 cycles CT \\
7 & DCL & 2 & $\mathrm{M}$ & 1 & $0 \mathrm{~mm}$ & None \\
\hline
\end{tabular}

$a$ : mm induration at $48 \mathrm{~h} ; b$ : cycle of 20 daily intramuscular injections of antimonial, $810 \mathrm{mg} \mathrm{Sb}(\mathrm{V})$ in adults: dose in children $20 \mathrm{mg} / \mathrm{kg} / \mathrm{d}$; $c$ : chemotherapy suspended after 2 cycles, severe adverse reactivity.

Case 2. Localized lesion on the right foot, followed 4 months later by development of an erythematous plaque on the nasal ala. Ulcer with infiltrated border, $25 \times 46 \mathrm{~mm}$ on the dorsum of the foot. Erythema and infiltration of the nasal ala, extending to the adjacent areas of the cheeks. Granuloma with epithelioid nodules and intracellular leishmanias.

Case 3. Antecedents: cutaneous lesion on the right arm 18 years earlier. Infiltration of right nasal ala extending to the nasal septum. Dense and diffuse macrophage granuloma with moderate epithelioid differentiation, leishmanias not observed.

Case 4. Antecedents: cutaneous lesion on arm treated with antimonials 33 years before presenting lesions in nasal fossa. Collapse of the bridge of the nose, loss of septal cartilage, infiltrated mucous membranes. Macrophage granuloma with abundant intracellular leishmanias.

Case 5. Nodular cutaneous lesions on thorax, left arm, right thigh. Abundant leishmanias in skin smears. Leishmanin reaction repeatedly negative. Undifferentiated macrophage granuloma with abundant leishmanias. Case 6. Initially presented ulcerated lesions on arms and face with abundant leishmanias in scrapings from border lesions. Subsequently developed nodular lesions, undifferentiated macrophage granuloma with abundant parasites. Transitory improvement with antimonials. Leishmanin reaction repeatedly negative.

Case 7. Extensive symmetrical plaques with abundant leishmanias on lower legs, spreading to arms and thorax. Leishmanin reaction repeatedly negative. Antimonials not administered. Macrophage granuloma with scarce epithelioid differentiation and abundant leishmanias.

Vaccine preparation and injection - L. (V.) braziliensis promastigotes, strain MHOM/BR/84/LTB 300, were cultivated for 3-4 days on Petri dishes containing blood agar base and $10 \%$ lysate of rabbit blood. After recovery and successive washes with phosphate-buffered saline (PBS), the parasites were counted in a Neubauer chamber and diluted to a concentration of $3 \times 10^{9} / \mathrm{ml}$. Parasites were killed by pasteurization in an Aguatherm shaking water bath, New Brunswick Scientific, at $56^{\circ} \mathrm{C}$ for $30 \mathrm{~min}$. Individual doses of $6 \times 10^{8}$ parasites $(0.2 \mathrm{ml}$ of the original 
suspension) were dispensed in sterile vials in a laminar flow hood. The Leishmania component of pasteurized vaccines was tested for complete parasite killing by direct observation with Trypan blue vital stain, cultivation on blood agar plates and injection in the footpads of normal and corticosteroid-suppressed BALB/c mice. Sterility was checked on by inoculation in BBL trypticase soy and Difco thioglycolate liquid media. Routine tests for toxicity, carried out by the intraperitoneal inoculation of one human dose/animal in groups of 5 mice and 2 guinea pigs and daily observation for 7 days for any clinical abnormality, were carried out at the National Institute of Hygiene of Venezuela, which also verified sterility as indicated above and certified each lot of vaccine. The vaccines were stored at $4^{\circ} \mathrm{C}$ until use.

Just before injection, BCG vaccine "SSI" (Mycobacterium bovis, Bacillus Calmette-Guerin, Danish strain 1331, Statens Serum Institut, Copenhagen, Denmark, lots 201005, 201040, and 201078) was suspended in diluted Sauton SSI solvent $\left(125 \mu \mathrm{g} \mathrm{MgSO}_{4}, 125 \mu \mathrm{g} \mathrm{K} \mathrm{HPO}_{4}, 1 \mathrm{mg}\right.$ L-asparagine $\cdot \mathrm{H}_{2} \mathrm{O}, 12.5 \mu \mathrm{g}$ iron ammonium citrate, 18.4

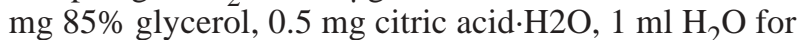
injection, manufacturer's insert) and $0.1 \mathrm{mg}$ of $\mathrm{BCG}$ (first dose) or $0.01 \mathrm{mg}$ BCG (successive doses) was added to each vial containing promastigotes. All doses were injected intradermally in the deltoid regions in two similar volumes of about $0.15 \mathrm{cc}$. Successive injections were separated by four-week intervals.

The vaccinations were carried out on an outpatient basis except for case 1 . This patient was hospitalized for four weeks because of his severe nutritional deficiency.

All patients were examined at two-week intervals or more frequently at the physicians' discretion.

Additional studies - Soluble extracts of fresh, autoclaved and pasteurized vaccines were prepared from a single concentrated suspension of promastigotes by rupture in an Aminco French pressure cell and subsequent centrifugation. The extracts were analyzed by electrophoresis of samples containing $50 \mu \mathrm{g}$ of protein on $10 \%$ polyacrylamide gel in the presence of sodium dodecyl sulphate (SDS-PAGE), as described by Laemmli (1970). Proteins were stained with Coomassie blue R250.

Skin smears were examined after Giemsa staining and biopsy specimens with were stained with hematoxylineosin.
Leishmanin was prepared in the Biochemistry Section, Institute of Biomedicine. Leishmanin skin tests were carried out as described previously, by the intradermal injection in the forearm of $0.1 \mathrm{ml}$ of an autoclaved suspension containing $6.25 \times 10^{6}$ L. (L.) pifanoi promastigotes $/ \mathrm{ml}$; induration was read at $48 \mathrm{~h}$ (Convit et al. 1989).

Relative concentrations of specific antibody $\mathrm{IgG}$ isotypes in DCL sera were determined in ELISA reactions using isotype-specific monoclonal antibodies against IgG1, 2, 3, and 4 (Biodesign International, Kennebunk, Maine, US) as described in detail previously (Ulrich et al. 1995). Briefly, microtiter plates sensitized with $2 \times 10^{7}$ formalin-fixed $L$. (L.) mexicana promastigotes/well were blocked and treated successively with a 1:100 dilution of patient's serum, 1:1200 dilutions of individual anti-isotype sera conjugated with peroxidase, and substrate. Positive and negative controls included in each assay were pools of DCL and normal sera, respectively.

\section{RESULTS}

All of the patients reported here showed complete remission of their lesions, leaving smooth scars on skin surfaces and smooth surfaces on mucosal membranes, with no persisting erythema, infiltration or detectable parasites. Remission has persisted for at least 10 months. The 3 patients with DCL developed positive indurated leishmanin reactions of 23,19 , and $14 \mathrm{~mm}$ at $48 \mathrm{~h}$ in patients 5, 6, and 7, respectively. The development of positive reactivity to leishmanin occurred simultaneously with ulceration of varying intensity in these patients. Lesions were resolved in MCL after 5 to 9 injections, while the early cases of DCL required 7 to 10 doses. A summary of time and number of vaccine doses to complete remission, duration of remission, final leishmanin reactivity and adverse reactions is presented in Table II. While all patients developed small ulcers at the sites of vaccine injection, only two reported fever or local pain which responded to non-prescription medication. Fig. 1 shows the response to immunotherapy of one of the patients with MCL, case 1, before and 6 months after the initiation of immunotherapy. The response of one of the patients with DCL (case 6) after 7 months is shown in Fig. 2.

Before initiation of the immunotherapy described here, specific anti-Leishmania antibodies of the IgG1 subclass predominated in the two youngest patients with DCL, 2

TABLE II

Summary of treatment with pasteurized vaccine in patients with severe mucocutaneous or early diffuse cutaneous leishmaniasis

\begin{tabular}{lccccc}
\hline Case & $\begin{array}{c}\text { Time of complete } \\
\text { remission }\end{array}$ & $\begin{array}{c}\text { Doses of } \\
\text { vaccine }\end{array}$ & $\begin{array}{c}\text { Duration of } \\
\text { remission }\end{array}$ & $\begin{array}{c}\text { Final } \\
\text { leishmanin }\end{array}$ & $\begin{array}{c}\text { Adverse } \\
\text { reactions }\end{array}$ \\
\hline 1 & 5 months & 5 & 2 months & $17 \mathrm{~mm}^{a}$ & $19 \mathrm{~mm}$ \\
2 & 5 months & 5 & 21 months & None & None \\
3 & 6 months & 6 & 11 months & $25 \mathrm{~mm}$ & Local pain, fever \\
4 & 9 months & 9 & 11 months & $28 \mathrm{~mm}$ & Fever \\
5 & 8 months & 9 & 12 months & $19 \mathrm{~mm}$ & None \\
6 & 10 months & 10 & 14 months & $14 \mathrm{~mm}$ & None \\
7 & 6 months & 7 & 14 months & None \\
\hline
\end{tabular}

a: $\mathrm{mm}$ induration at $48 \mathrm{~h}$ 

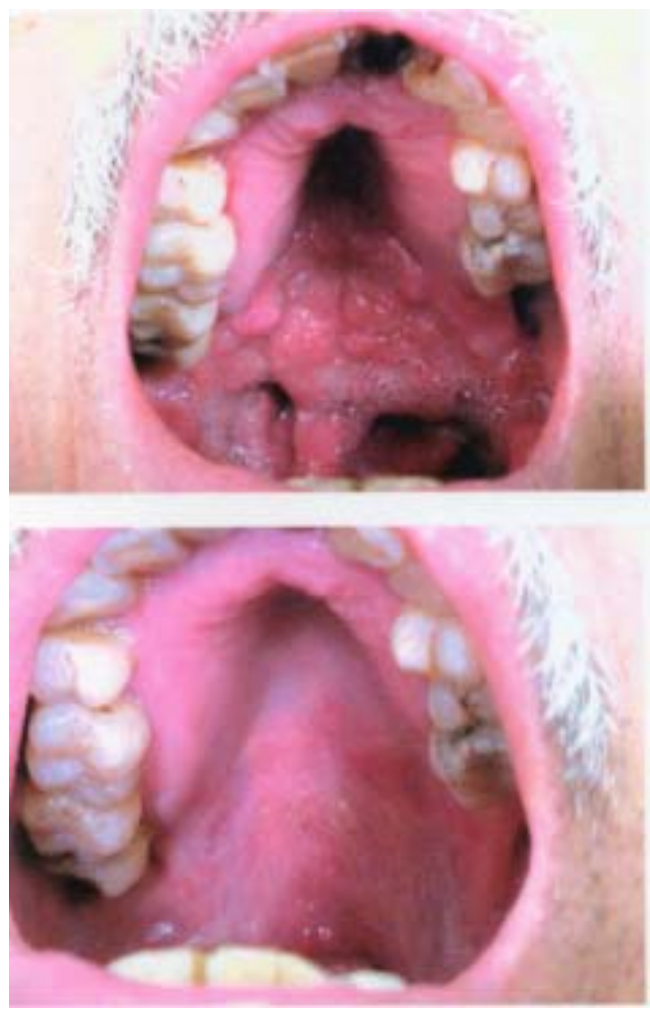

Fig. 1: patient, case 1, with mucocutaneous leishmaniasis showing lesions of the palate, uvula, and pharynx before (above) and 6 months after (below) immunotherapy was begun.
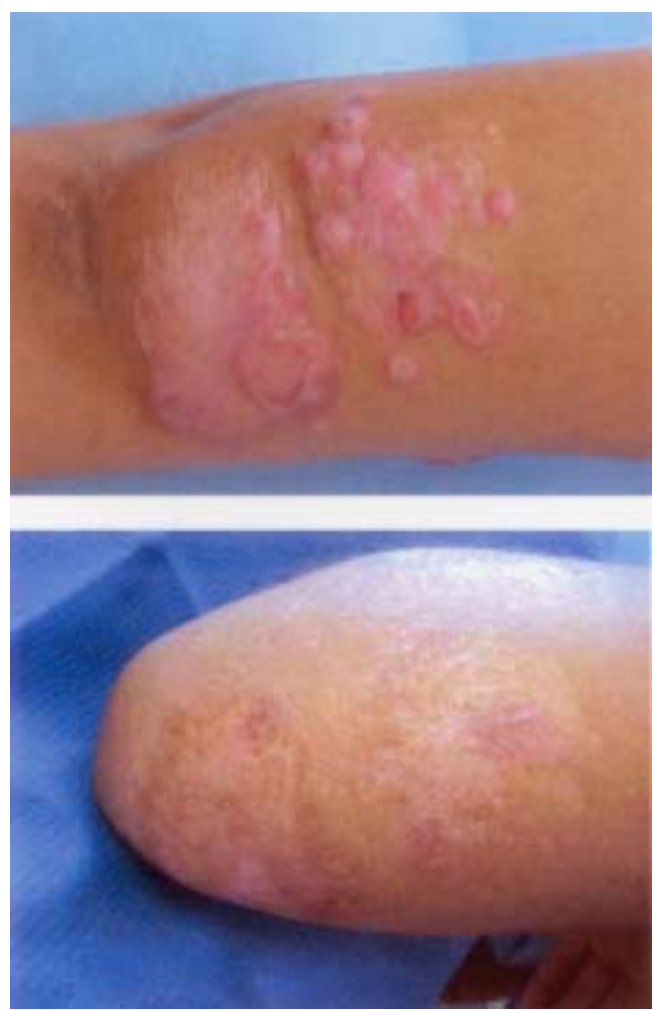

Fig. 2: extensive cutaneous plaques of early diffuse cutaneous leishmaniasis, case 6, before (above) and 7 months after (below) immunotherapy. and 6 years old, cases 5 and 7; the predominant IgG subclass in the 10-year-old, case 6, was IgG4. Immunotherapy was not accompanied by significant changes in the isotype distribution of specific antibodies during an average one-year observation period (data not shown).

Fig. 3 shows the SDS-PAGE analysis of extracts from pasteurized, autoclaved and fresh Leishmania promastigotes. Using sample sizes with similar protein content, the integrity of proteins in the extract from the pasteurized sample was essentially unaltered when compared to the extract from fresh promastigotes, as indicated by the notable similarity in the intensity and relative mobility of more than 25 distinct bands, widely distributed throughout the entire range of about 15 to $120 \mathrm{kDa}$. In marked contrast, a single well-defined band of about $72 \mathrm{kDa}$ and weak diffuse bands in the 15 to $90 \mathrm{kDa}$ range were observed in the extract of the autoclaved promastigote suspensions.

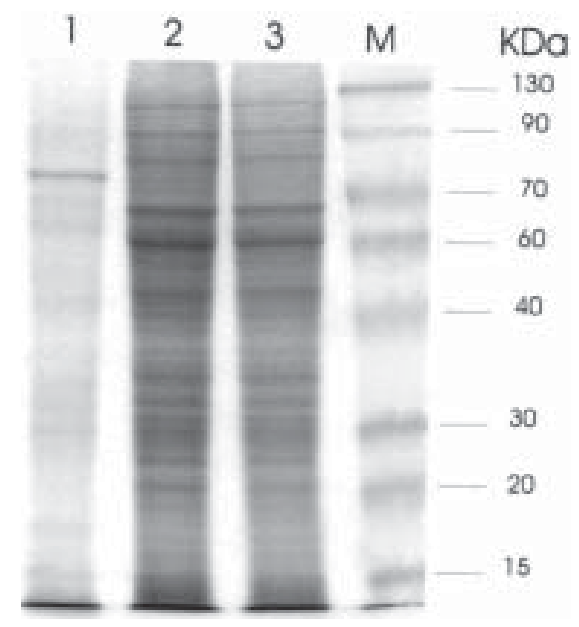

Fig. 3: separation by electrophoresis on a sodium dodecyl sulphatepolyacrylamide gel $(10 \%)$ of samples containing $50 \mu \mathrm{g}$ of soluble protein from autoclaved (lane 1), fresh (lane 2) and pasteurized $L$. (V.) braziliensis LTB 300. Gibco-BRL molecular weight markers are shown in lane $\mathrm{M}$, with corresponding $\mathrm{kDa}$ to the right. The gel was stained with Coomassie blue R250.

\section{DISCUSSION}

It has long been recognized that some forms of ACL are refractory to treatment with meglumine antimoniate or other antimonial derivatives. One of these, DCL, may show a transitory response to chemotherapy. Becker et al. (1999) observed apparent parasitological cures after treatment of two DCL patients with pentamidine, allopurinol and IFN-gamma, but relapse began three months after suspending treatment, in spite of the re-establishment of conditions favoring a Th1 response (Salaiza-Suazo et al. 1999). Transitory clinical improvement induced by treatment has been associated with expression of IFN-gamma mRNA, but relapse was not prevented (Bomfim et al. 1996).

Mucosal leishmaniasis frequently responds favorably to treatment with antimonial derivatives, but refractory cases have been reported. Lessa et al. (2001) reported 
clinical cure in 10 patients with refractory disease when pentoxifylline, a tumor necrosis alpha inhibitor, was associated with pentavalent antimony. Badaró et al. (2001) also reported successful treatment of a case of refractory mucosal leishmaniasis using a mixture of 4 Leishmania recombinant antigens together with GM-CSF adjuvant.

Earlier studies have shown that the spectrum of human cutaneous leishmaniasis, particularly with regard to the 2 polar forms, reflects immunological responses which are quite similar to those reported in murine models of the disease, which have been fundamental in defining Th1 and Th2 subpopulations of lymphocytes (Mosmann et al. 1986). The LCL and DCL forms of human disease are characterized by relatively predominant $\mathrm{TH} 1$ and $\mathrm{Th} 2$ immune responses, respectively, although small amounts of the non-predominant cytokines are present (CáceresDittmar et al. 1993, Pirmez et al. 1993, Convit et al. 1993). ICL, including cutaneous and muco-cutaneous manifestations, presents a complex response characterized by the presence of TH1 and TH2 cytokines as well as other components such as TGF- $\beta$ and interleukin-10 in lesions (Cáceres-Dittmar et al. 1993, Díaz et al. 2002, Bacellar et al. 2002).

We have used immunotherapy in ACL for more than a decade with several objectives in mind. These include the search for an effective, acceptable, safe, and economical alternative to the use of relatively toxic antimonial derivatives in the treatment of the disease. A primary objective has been to induce or reinforce a persisting Th1 response, facilitating healing without significant side effects. Immunotherapy with a combined vaccine containing autoclaved L. (L.) amazonensis promastigotes and viable BCG has been shown to induce a Th1-like response in patients with ACL (Cabrera et al. 2000). BCG, used in the combined vaccine as an adjuvant, has been used in millions of individuals, including newborn infants, with remarkably few side-effects when administered in the appropriate dose by the intradermal route. Adverse effects including excessively large ulcerations or lymphangenitis are more likely to be associated with subcutaneous injection, excessive dose or pre-existing hypersensitivity and have been infrequent in our experience, even after numerous vaccinations. Minimal side effects have been reported using 2 doses of prophylactic vaccine containing BCG in Sudan (Khalil et al. 2000), but in Colombia adverse reactions were sufficiently severe to merit suspension of a third dose (Velez et al. 2000). BCG has been clearly associated with the induction of a Th1 immune response (Tokunga et al. 1999) and is probably the most acceptable TH1-inducing adjuvant presently available for use in humans. While immunotherapy with BCG and autoclaved promastigotes of $L$. (L.) amazonensis is associated with clinical cure in more than $95 \%$ of Venezuelan patients with uncomplicated LCL (Convit et al. 1989, 2003), it is less effective in intermediate forms of the disease and ineffective, except for transitory improvement, even when combined with chemotherapy in some of these patients and in those suffering from DCL.

Early unpublished field studies by our group demonstrated that vaccination with autoclaved L. (V.) braziliensis-BCG induced significantly stronger leishmanin reactivity than autoclaved $L$. (L.) amazonensis-BCG. However, the principal cause of ACL in Venezuela at that time was considered to be $L$. (L.) mexicana, which was much easier to cultivate in large quantities. Subsequent large-scale epidemiological studies have shown that infection by L. (V.) braziliensis is, in reality, more frequent throughout the country (Rodríguez et al. 2002). Preliminary studies in guinea pigs demonstrated that the vaccine described in this paper was innocuous and immunogenic, as measured by positive leishmanin skin tests and rapid involution of lesions after challenge with $L$. $(L$.) enriettii compared to control animals (unpublished data). Therefore the decision was made to alter the Leishmania component of the combined vaccine. In addition, the milder conditions of parasite killing by pasteurization may conserve a significantly larger group of immunogenic epitopes, reflected indirectly by no detectable alteration in the pattern of protein components observed in SDSPAGE analysis.

In Venezuela, DCL is a disease which apparently usually begins during childhood, but diagnosis is often not made until many years later. Leishmanin reactivity is persistently negative in these patients, even during periods when chemotherapy induces transitory clinical improvement. The three patients with early DCL described here all developed delayed-type hypersensitivity to Leishmania during the course of immunotherapy, reflected in positive skin tests averaging $18.7 \mathrm{~mm}$ of induration.

Several years ago, we reported that nearly all patients (19/20) with DCL of long duration produce specific antiLeishmania antibodies of the IgG4 isotype, compatible with a Th2 response (Ulrich et al. 1995). In the 2 youngest of the 3 patients reported here, a predominant IgG1 Leishmania-specific response was observed before treatment was begun and persisting after remission. This could partially reflect the fact that the maturation of the IgG4 subclass is particularly slow in children (Lee et al. 1986). Barral et al. (1995) have described a spectrum within the DCL group in Brazil, including subpolar cases with negative leishmanin reactions and distinctive histopathological characteristics. The clinical and particularly the histopathological characteristics of the three patients in this study are not characteristic of subpolar DCL as described by these authors.

Compassionate need has been a primary motivation of the use of this vaccine therapy in a small group of severely affected patients with little or no hope of control of their disease in this preliminary study. We fully expect this form of immunotherapy to supplant the previously used preparation with the autoclaved Leishmania component, but phases of protection in animals and longterm follow-up in early clinical trials are still underway. We recognize that the observation period in this group of patients is relatively short in terms of the long-term manifestations of mucosal and diffuse cutaneous leishmaniasis. We consider that booster injections would be useful if follow-up shows weakening of leishmanin reactivity in patients with DCL or if any clinical evidence of reactivation is observed in patients with MCL.

The favorable clinical response in severe forms of leishmaniasis without significant side-effects suggests that 
the vaccine described here might be useful in immunotherapy of more common forms of cutaneous leishmaniasis and in vaccination against the disease.

\section{REFERENCES}

Bacellar O, Lessa H, Schiefer A, Machado P, Ribeiro de Jesus A, Dutra WO, Gollob KJ, Carvalho EM 2002. Up-regulation of Th-1 responses in mucosal leishmaniasis patients. Infect Immun 70: 6734-6740.

Badaró R, Lobo I, Nakatani M, Muinos A, Netto EM, Coler RN, Reed SG 2001. Successful use of a defined antigen/ GM-CSF adjuvant vaccine to treat mucosal leishmaniasis refractory to antimony: a case report. Braz J Infect Dis 5: 223-232.

Barral A, Costa JM, Bittencourt AL, Barral-Netto M, Carvalho EM 1995 Polar and subpolar diffuse cutaneous leishmaniasis in Brazil: clinical and immunopathologic aspects. Int J Dermatol 34: 474-479.

Becker I, Volkow P, Velasco-Castrejon O, Salaiza-Suazo N, Berzunza-Cruz M, Domínguez JS, Morales-Vargas A, RuizRemigio A, Pérez-Montfort R 1999. The efficacy of pentamidine combined with allopurinol and immunotherapy for the treatment of patients with diffuse cutaneous leishmaniasis. Parasitol Res 85: 165-170.

Bomfim G, Nascimento C, Costa J, Carvalho EM, Barral-Netto M, Barral A 1996. Variation of cytokine patterns related to therapeutic response in diffuse cutaneous leishmaniasis. Exp Parasitol 84: 188-194.

Cabrera M, Blackwell JM, Castes M, Trujillo D, Convit J, Shaw MA 2000. Immunotherapy with live BCG plus heat killed Leishmania induces a $\mathrm{T}$ helper 1-like response in American cutaneous leishmaniasis patients. Parasite Immunol 22: 73-79.

Cáceres-Dittmar, G, Tapia FJ, Sánchez NA, Yamamura M, Uyemura K, Modlin RL, Bloom BR, Convit J 1993. Determination of the cytokine profile in American cutaneous leishmaniasis using the polymerase chain reaction. Clin Exp Immunol 91: 500-505.

Carvalho EM, Barral A, Costa JM, Bittencourt A, Marsden P 1994. Clinical and immunopathological aspects of disseminated cutaneous leishmaniasis. Acta Trop 56: 315-325.

Convit J, Castellanos PL, Rondón AJ, Pinardi ME, Ulrich M, Castés M, Bloom B, García L 1987. Immunotherapy versus chemotherapy in localised cutaneous leishmaniasis. The Lancet Feb 21: 401-404.

Convit J, Castellanos PL, Ulrich M, Castés M, Rondón A, Pinardi ME, Rodríguez N, Bloom BR, Formica S, Valecillos L, Bretaña A 1989. Immunotherapy of localized, intermediate and diffuse forms of American cutaneous leishmaniasis. J Infect Dis 160: 104-115.

Convit J, Ulrich M, Fernández CT, Tapia FJ, Cáceres-Dittmar G, Castés M, Rondón AJ 1993. The clinical and immunological spectrum of American cutaneous leishmaniasis. Trans R Soc Trop Med Hyg 87: 444-448.

Convit J, Ulrich M, Zerpa O, Borges R, Aranzazu N, Valera M, Villarroel H, Zapata Z, Tomedes I 2003. Immunotherapy of American cutaneous leishmaniasis in Venezuela, 1990 to 1999. Trans $R$ Soc Trop Med Hyg in press.

Díaz NL, Zerpa O, Ponce LV, Convit J, Rondón AJ, Tapia FJ 2002. Intermediate or chronic cutaneous leishmaniasis: leukocyte immunophenotypes and cytokine characterization of the lesion. Exp Dermatol 11: 34-41.

Grimaldi Jr G, Tesh RB, McMahon-Pratt D 1989. A review of the geographic distribution and epidemiology of leishmaniasis in the New World. Am J Trop Med Hyg 41: 687-725.

Khalil EA, Elhassan AM, Zilstra EE, Osman OF, Eljack IA, Ibrahim ME, Mukhtar MM, Ghalib HW, Modabber F 2000. Safety and immunogenicity of an autoclaved Leishmania major vaccine. East Africa Med J 77: 468-470.

Laemmli UK 1970. Cleavage of structural proteins during the assembly of the head of bacteriophage T4. Nature Aug 15, 227(259): 680-685.

Lee SI, Heiner DC, Wara D 1986. Development of serum IgG subclass levels in children. Monogr Allergy 19: 108.

Lessa HA, Machado P, Lima F, Cruz AA, Bacellar O, Guerreira J, Carvalho EM 2001. Successful treatment of refractory mucosal leishmaniasis with pentoxifylline plus antimony. Am J Trop Med Hyg 65: 87-89.

Mosmann TR, Cherwinski H, Bond MW, Giedlin MA, Coffman RL 1986. Two types of murine helper T cell clone. I. Definition according to profiles of lymphokine activities and secreted proteins. J Immunol 136: 2348-2357.

Pirmez C, Yamamura M, Uyemura K, Paes-Oliveira M, Conceição-Silva F, Modlin R 1993. Cytokine patterns in the pathogenesis of human leishmaniasis. J Clin Invest 91: $1390-1395$

Reiner SL, Locksley RM 1993. Cytokines in the differentiation of Th1/Th2 CD4+ subsets in leishmaniasis. J Cell Biochem 53: 323-328.

Rodríguez N, De Lima H, Aguilar CM, Rodríguez A, Barker DC, Convit J 2002. Molecular epidemiology of cutaneous leishmaniasis in Venezuela. Trans R Soc Trop Med Hyg 96 (Suppl. 1): S105-S109.

Salaiza-Suazo N, Volkow P, Tamayo R, Moll H, Gillitzer R, Pérez-Torres A, Pérez-Montfort R, Domínguez JD, VelascoCastrejon O, Crippa M, Becker I 1999. Treatment of two patients with diffuse cutaneous leishmaniasis caused by Leishmania mexicana modifies the immunohistological profile but not the disease outcome. Trop Med Int Hlth 4: 801811.

Tokunga T, Yamamoto T, Yamamoto S 1999. How BCG led to the discovery of immunostimulatory DNA. Jap J Infect Dis 52: 1-11.

Ulrich M, Rodríguez V, Centeno M, Convit J 1995. Differing antibody $\mathrm{IgG}$ isotypes in the polar forms of leprosy and cutaneous leishmaniasis characterized by antigen- specific T cell anergy. Clin Exp Immunol 100: 54-58.

Velez ID, del Pilar Agudelo S, Arbelaez MP, Gilchrist K, Robledo SM, Puerta JA, Zicker F, Berman J, Modabber 2000. Safety and immunogenicity of a killed Leishmania (L.) amazonensis vaccine against cutaneous leishmaniasis in Colombia: A randomized controlled trial. Trans R Soc Trop Med Hyg 94: 698-703. 